\title{
A simulação realística como ferramenta de aprendizagem para a Sistematização da
}

\section{Assistência de Enfermagem}

\author{
A realistic simulation as a learning tool for the Systematization of Nursing Care \\ La simulación realista como herramienta de aprendizaje para la Sistematización de la Atención de \\ Enfermería
}

Recebido: 29/06/2021 | Revisado: 05/07/2021 | Aceito: 08/07/2021 | Publicado: 22/07/2021

\author{
Larissa das Neves Castro \\ ORCID: https://orcid.org/0000-0001-7592-6236 \\ Instituto Brasileiro de Medicina e Reabilitação, Brasil \\ E-mail: castro97rj@gmail.com \\ Camylla Mendonça Santiago \\ ORCID: https://orcid.org/0000-0003-3030-8121 \\ Universidade do Estado do Rio de Janeiro, Brasil \\ E-mail: myllamendonca96@gmail.com \\ Bheatriz da Costa Diniz Olegário \\ ORCID: https://orcid.org/0000-0001-5969-8894 \\ Universidade do Estado do Rio de Janeiro, Brasil \\ E-mail: bheatriz789@gmail.com \\ Julia Negrato Cardoso \\ ORCID: https://orcid.org/0000-0002-6788-490X \\ Universidade do Estado do Rio de Janeiro, Brasil \\ E-mail: julianegrato@gmail.com \\ Hanna Oliveira Temperini \\ ORCID: https://orcid.org/0000-0003-4432-2098 \\ Universidade do Estado do Rio de Janeiro, Brasil \\ E-mail: hannatemperini@ outlook.com \\ Advi Catarina Barbachan Moraes \\ ORCID: https://orcid.org/0000-0002-1470-2623 \\ Universidade do Estado do Rio de Janeiro, Brasil \\ E-mail: advicbmoraes@gmail.com \\ Alessandra Sant Anna Nunes \\ ORCID: https://orcid.org/0000-0001-7435-2568 \\ Universidade do Estado do Rio de Janeiro, Brasil \\ E-mail: asantnunes@gmail.com
}

\begin{abstract}
Resumo
Objetivo: Analisar a percepção dos discentes acerca da aplicabilidade da Sistematização da Assistência de Enfermagem (SAE) no cenário de simulação realística e como esse processo contribui no aprendizado do acadêmico. Método: Revisão integrativa da literatura sobre o uso da simulação realística como ferramenta para a aprendizagem da sistematização da assistência em enfermagem. Para maior assertividade metodológica a seguinte pergunta PICO foi utilizada: O treinamento da Sistematização da Assistência de Enfermagem através da simulação realística traz melhores resultados na aprendizagem dos discentes de enfermagem comparado aos métodos tradicionais de ensino? Os dados bibliográficos do estudo foram levantados de maneira eletrônica, através da BVS, os descritores utilizados foram: "Treinamento por simulação" e "Estudantes de enfermagem", com operados boleano "AND". Resultados: Foram analisados 8 estudos, sendo eles: transversais, quase-experimentais, experimentais randomizados e pesquisas de intervenção, no intervalo entre 2016 a 2021, nos idiomas inglês e português. randomizado do ano de 2016 a 2021 , em inglês e português. Em todos os artigos verificados a simulação realística é tida como um importante método para o ensino para o desenvolvimento da Sistematização da Assistência de Enfermagem. Conclusão: O estudo pôde concluir que os estudantes, após os cenários, estavam cientes de seus erros antes de receber o feedback de seus preceptores. Portanto, a simulação realística pode ser uma boa alternativa para facilitar a segurança, evitar erros e solidificar a aprendizagem da sistematização da assistência de enfermagem.
\end{abstract}

Palavras-chave: Estudantes de enfermagem; Simulação realística; Sistematização da assistência; Ensino.

\footnotetext{
Abstract

Objective: To analyze the perception of students about the applicability of the Systematization of Nursing Care (SAE) in the realistic simulation scenario and how this process contributes to academic learning. Method: Integrative literature review on the use of realistic simulation as a tool for learning the systematization of nursing care. For
} 
greater methodological assertiveness, the following PICO question was used: Does training in the Systematization of Nursing Care through realistic simulation bring better results in the learning of nursing students compared to traditional teaching methods? The bibliographic data of the study were collected electronically, through the VHL, the descriptors used were: "Training by simulation" and "Nursing students", with Boolean operations "AND". Results: Eight studies were analyzed, namely: cross-sectional, quasi-experimental, randomized experimental and intervention research, in the interval between 2016 and 2021, in English and Portuguese. randomized from 2016 to 2021, in English and Portuguese. In all articles verified, realistic simulation is seen as an important teaching method for the development of Nursing Care Systematization. Conclusion: The study could conclude that students, after the scenarios, were aware of their mistakes before receiving feedback from their preceptors. Therefore, realistic simulation can be a good alternative to facilitate safety, avoid errors and solidify the learning of nursing care systematization.

Keywords: Nursing students; Realistic simulation; Care systematization; Teaching.

\section{Resumen}

Objetivo: Analizar la percepción de los estudiantes sobre la aplicabilidad de la Sistematización de la Atención de Enfermería (SAE) en el escenario de simulación realista y cómo este proceso contribuye al aprendizaje académico. Método: revisión integradora de la literatura sobre el uso de la simulación realista como herramienta para el aprendizaje de la sistematización del cuidado de enfermería. Para una mayor asertividad metodológica se utilizó la siguiente pregunta PICO: ¿La formación en la Sistematización de la Atención de Enfermería a través de la simulación realista trae mejores resultados en el aprendizaje de los estudiantes de enfermería en comparación con los métodos tradicionales de enseñanza? Los datos bibliográficos del estudio fueron recolectados electrónicamente, a través de la BVS, los descriptores utilizados fueron: "Capacitación por simulación" y "Estudiantes de enfermería", con operaciones booleanas "Y". Resultados: Se analizaron ocho estudios, a saber: investigación transversal, cuasiexperimental, experimental aleatorizada y de intervención, en el intervalo entre 2016 y 2021, en inglés y portugués. aleatorizados de 2016 a 2021, en inglés y portugués. En todos los artículos verificados, la simulación realista es vista como un método de enseñanza importante para el desarrollo de la Sistematización del Cuidado de Enfermería. Conclusión: El estudio pudo concluir que los estudiantes, luego de los escenarios, eran conscientes de sus errores antes de recibir retroalimentación de sus preceptores. Por tanto, la simulación realista puede ser una buena alternativa para facilitar la seguridad, evitar errores y solidificar el aprendizaje sobre la sistematización del cuidado de enfermería.

Palabras clave: Estudiantes de enfermería; Simulación realista; Sistematización asistencial; Enseñanza.

\section{Introdução}

A Simulação Realística é uma metodologia de treinamento inovadora responsável por auxiliar o estudante no seu processo de formação profissional com estratégias para o desenvolvimento de habilidades técnicas e não técnicas, tendo em vista o crescimento do seu conhecimento. As habilidades técnicas são compostas de processos específicos de cada especialidade, já as não técnicas englobam as habilidades cognitivas e sociais que complementam a técnica para a atuação da prática do profissional com qualidade e segurança. Sua metodologia expõe situações comuns e semelhantes do ambiente de trabalho através de um cenário, apresentando diferentes graus de complexidade, além de estimular o trabalho em equipe entre os estudantes, a relação enfermeiro-paciente e o pensamento crítico e de liderança. (Bellaguarda, et al., 2020).

Essa nova metodologia de ensino ganhou espaço nas graduações em saúde mediante as observações positivas no meio de experiências relatadas através dos ensinamentos em simulações, com isso gerando cada vez mais a necessidade de alinhar os conteúdos teórico-práticos. Essa estratégia de aprendizagem vem sendo de extrema importância para que os estudantes construam um pensamento crítico e raciocínio lógico antes de chegarem ao mercado de trabalho. Constatando assim que o método tradicional de ensino, o qual é feito através de aula expositiva, pode ser mais eficaz em conjunto com métodos mais inovadores. (Ferreira, et al., 2018).

O presente estudo aborda a utilização da simulação realística como ferramenta de ensino para a sistematização da assistência de enfermagem, através do levantamento de pesquisas bibliográficas foi buscado se o ensino da SAE através da simulação realística tem gerado efeitos positivos comparados aos métodos tradicionais.

Baseando-se no contexto histórico, a enfermagem possui uma trajetória que vem incorporando, gradualmente, à sua prática ações pautadas em metodologias que lhe confiram cada vez mais caráter científico e confiabilidade clínica. No qual, em 
1970, a enfermeira Wanda Horta, desenvolveu no Brasil o Processo de Enfermagem, o que basificou a Sistematização da Assistência em Enfermagem, levando em consideração todo o histórico apresentado pelo paciente, suas dificuldades individuais, diagnósticos específicos e intervenções programadas para cada diagnóstico atribuído.

No ano de 2009, através da Resolução n. 358, o Conselho Federal de Enfermagem (COFEN) introduziu a Sistematização da Assistência em Enfermagem como atividade inerente do Enfermeiro, onde o mesmo necessita realizar uma análise prévia do quadro clínico do paciente para elaborar a prescrição de cuidado. Tal ato normativo permitiu que a assistência de enfermagem atravesse a barreira dos cuidados exclusivamente médicos e se torne peça chave no cuidado integral ao paciente, com autonomia, conhecimentos técnico-científicos e abordagem clínica. Sendo assim, por meio deste avanço, o processo de trabalho torna-se mais organizado em virtude da aplicação do Processo de Enfermagem (PE) e suas etapas que auxiliam na construção de um pensamento crítico. (Meneses, et al., 2019)

Para entender melhor os motivos que levam a inserção da simulação realística no ensino e aprendizagem da sistematização em enfermagem, tem se como pergunta norteadora deste artigo: O treinamento da Sistematização da Assistência de Enfermagem através da simulação realística traz melhores resultados na aprendizagem dos discentes de enfermagem comparado aos métodos tradicionais de ensino?

A partir da realização prévia de procedimentos e a possibilidade de erros que o aluno poderá cometer em um cenário de simulação, destaca-se a vantagem da metodologia fundamentada em simulação por gerar um aprendizado seguro ao aluno. Mediante aos erros o aluno tem a possibilidade de aprender com os mesmos e refazer o procedimento até que se sinta seguro, refletindo assim na sua prática ao não cometê-los, por tanto tendo um aprendizado sem pôr a vida de alguém em risco. Além do incentivo ao raciocínio crítico, e auxílio na resolução de problemas. (Alves, et al., 2019).

De acordo com a temática do trabalho, usamos as palavras chaves "Treinamento por simulação" e "Estudantes de Enfermagem" para a busca de artigos que nos darão embasamento para falar a respeito do objetivo da pesquisa, a qual tem como finalidade analisar a percepção dos discentes acerca da aplicabilidade da Sistematização da Assistência de Enfermagem (SAE) no cenário de simulação realística e como esse processo contribui no aprendizado do acadêmico.

\section{Metodologia}

Trata-se de uma revisão integrativa de literatura, norteada pelos parâmetros de José Carlos Köche (2011). Segundo Köche, uma revisão de literatura tem como objetivo fundamentar e expandir o conhecimento dos investigadores, baseando sua análise em contribuições teóricas já produzidas.

Para maior assertividade metodológica foi empregada a estratégia PICO (acrônimo para Paciente, Intervenção, Comparação e Outcome/Desfecho) para basificar a confecção deste artigo, a partir disso a seguinte pergunta foi estabelecida: "O treinamento da Sistematização da Assistência de Enfermagem através da simulação realística traz melhores resultados na aprendizagem dos discentes de enfermagem comparado aos métodos tradicionais de ensino?". A base de dados utilizada foi a BVS (Biblioteca Virtual em Saúde), acessada durante o período de 7 a 14 de Janeiro de 2021.

O levantamento bibliográfico transcorreu de maneira virtual, utilizando os descritores, escolhidos através do sistema DeCS/MeSH, "Treinamento por simulação" e "Estudantes de enfermagem", com operador booleano "AND". Após registrar o total de artigos encontrados (436), os seguintes critérios de inclusão foram aplicados, respectivamente: últimos cinco anos, idiomas inglês, português e espanhol e todos os tipos de estudos, exceto os de revisão sistemática, como resultado foram encontrados respectivamente 346, 340 e por fim 91 artigos. A Figura 1 descreve, em forma de fluxograma, os critérios de inclusão e exclusão aplicados. 
Figura 1: Fluxograma dos critérios de inclusão e exclusão.

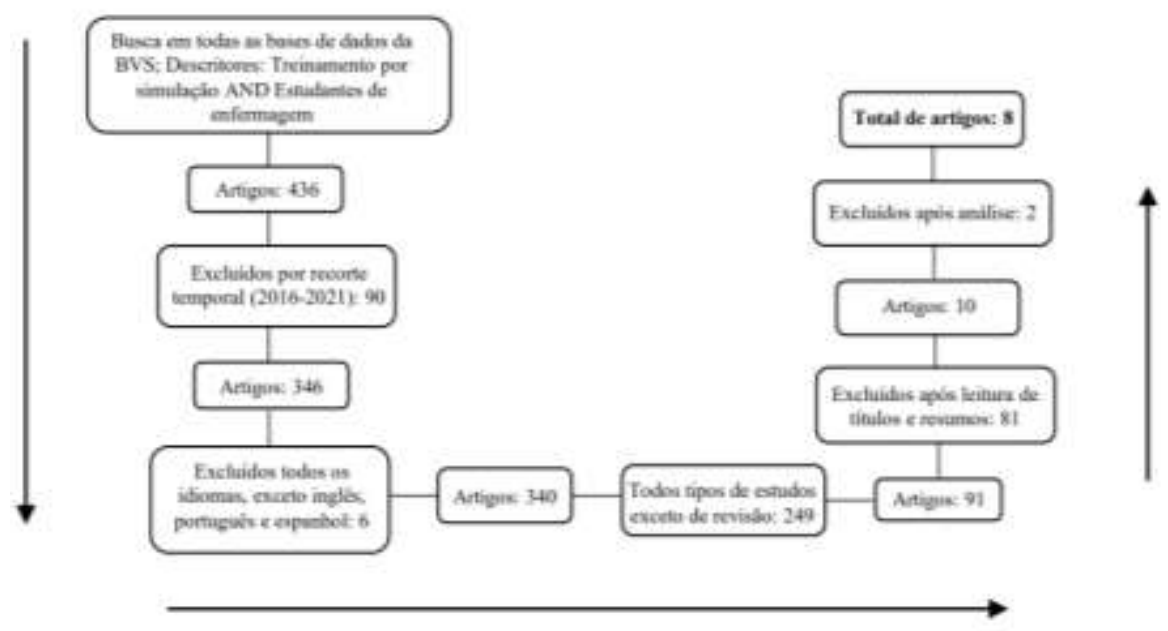

Fonte: Autores (2021).

Foram selecionados os estudos que respondiam ao objetivo central da revisão, ou seja, os artigos que abordassem a simulação realística e seus resultados em comparação aos métodos tradicionais de ensino e o treinamento da Sistematização da Assistência em Enfermagem (SAE) através da simulação. A maioria dos artigos não contemplava a SAE de forma integral, sendo assim, foram escolhidos aqueles que traziam alguma etapa do processo de enfermagem ou que em seu título ou resumo citassem acerca do pensamento crítico, raciocínio clínico, tomada de decisão e intervenções. Houve duas reuniões, a primeira para estabelecer os filtros e a segunda para definir os artigos escolhidos. A escolha dos estudos foi estabelecida por pares, onde cada dupla selecionava e analisava em conjunto, se o estudo poderia ou não ser incorporado ao artigo. Após essa verificação, determinou-se que 8 dissertações seriam efetivamente investigadas. A Tabela 1 elucida os parâmetros observados, ano do estudo, país e quantidade de estudantes envolvidos 
Tabela 1: Enquadramento dos artigos para análise.

\begin{tabular}{|c|c|c|c|c|c|}
\hline Código & Título & Ano & País & Tipo de estudo & $\begin{array}{l}\quad \mathrm{N}^{\circ} \text { de } \\
\text { estudantes } \\
\text { envolvidos }\end{array}$ \\
\hline 1 & $\begin{array}{c}\text { Aspectos positivos e negativos da simulação clínica no ensino } \\
\text { de enfermagem }\end{array}$ & 2020 & Brasil & $\begin{array}{c}\text { Estudo transversal } \\
\text { descritivo de abordagem } \\
\text { qualitativa }\end{array}$ & 36 \\
\hline 2 & $\begin{array}{c}\text { Simulação realística e seus atributos para a formação do } \\
\text { enfermeiro }\end{array}$ & 2019 & Brasil & $\begin{array}{l}\text { Estudo quantitativo, } \\
\text { transversal e analítico }\end{array}$ & 55 \\
\hline 3 & $\begin{array}{l}\text { Comparison of the effects of simulation training and problem- } \\
\text { based scenarios on the improvement of graduating nursing } \\
\text { students to speak up about medication errors: A quasi- } \\
\text { experimental study }\end{array}$ & 2020 & Taiwan & Estudo quase-experimental & 93 \\
\hline 4 & $\begin{array}{l}\text { Autoconfiança e satisfação dos estudantes de enfermagem em } \\
\text { simulação de emergência }\end{array}$ & 2020 & Brasil & $\begin{array}{l}\text { Pesquisa de intervenção, de } \\
\text { braço único, longitudinal do } \\
\text { tipo antes e depois }\end{array}$ & 35 \\
\hline 5 & $\begin{array}{l}\text { Desenvolvimento da competência de avaliação clínica do } \\
\text { paciente crítico por acadêmicos de enfermagem: Contribuição } \\
\text { da Simulação }\end{array}$ & 2020 & Brasil & $\begin{array}{l}\text { Estudo qualitativo e } \\
\text { analítico }\end{array}$ & 14 \\
\hline 6 & $\begin{array}{l}\text { Comparing the Effects of Simulation-Based and Traditional } \\
\text { Teaching Methods on the Critical Thinking Abilities and Self- } \\
\text { Confidence of Nursing Students }\end{array}$ & 2018 & $\begin{array}{l}\text { Arábia } \\
\text { Saudita }\end{array}$ & Experimental & 30 \\
\hline 7 & $\begin{array}{l}\text { Impact of prebriefing on competency performance, clinical } \\
\text { judgment and experience in simulation: An experimental study }\end{array}$ & 2016 & Canadá & Experimental randomizado & 76 \\
\hline 8 & $\begin{array}{c}\text { Effectiveness of Integrated Simulation and Clinical } \\
\text { Experiences Compared to Traditional Clinical Experiences for } \\
\text { Nursing Students }\end{array}$ & 2016 & $\begin{array}{l}\text { Estados } \\
\text { Unidos }\end{array}$ & Experimental & 124 \\
\hline
\end{tabular}

Fonte: Autores (2021).

\section{Resultados e Discussão}

O uso da Simulação é considerado um importante método para o ensino de estudantes de enfermagem, pois permite que os mesmos coloquem em prática procedimentos e situações prováveis antes de realizá-los em um ambiente real. Os estudos analisados evidenciam que a SAE, principalmente na etapa de "Intervenção", consegue ser aprimorada durante as práticas realísticas controladas. Na Tabela 2 observa-se os resultados obtidos em cada artigo. 
Tabela 2: Resultados obtidos em cada estudo analisado.

\begin{tabular}{|c|c|c|c|}
\hline Código & Ano & País & Resultados \\
\hline 1 & 2020 & Brasil & $\begin{array}{c}\text { Aspectos positivos da Simulação Realística segundo o estudo: possibilita a preparação técnica e psicológica } \\
\text { de discentes para a prática clínica, a correlação entre teoria e prática e o desenvolve raciocínio crítico. } \\
\text { Aspecto negativo da Simulação Realística segundo o estudo: nervosismo dos discentes ao realizar as } \\
\text { atividades propostas na simulação }\end{array}$ \\
\hline 2 & 2019 & Brasil & $\begin{array}{c}\text { Este estudo identificou diferenças entre os discentes do quarto e do nono período no que se refere a } \\
\text { percepção da aplicação da Sistematização da Assistência de Enfermagem. Os estudantes do quarto período } \\
\text { obtiveram uma percepção mais positiva de que a simulação realística desenvolve competências e } \\
\text { conhecimentos teóricos-científicos. }\end{array}$ \\
\hline 3 & 2020 & Taiwan & $\begin{array}{c}\text { Esse estudo observou que os alunos mudaram suas atitudes em relação à segurança dos medicamentos após } \\
\text { os cenários de simulação e se sentiram mais relaxados para conversarem sobre os erros, pois eles } \\
\text { subestimavam as chances de que erros poderiam ocorrer devido à insegurança para falar sobre esses } \\
\text { potenciais erros. }\end{array}$ \\
\hline 4 & 2020 & Brasil & $\begin{array}{l}\text { A pesquisa mostrou aumento da autoconfiança dos discentes relacionada à avaliação e intervenção em casos } \\
\text { clínicos simulados de emergência, como em uma PCR, e foi constatado que os alunos tiveram maior grau de } \\
\text { satisfação com seus aprendizados. Foi observado que ao longo do tempo a simulação prepara e dá suporte ao } \\
\text { futuro enfermeiro. Esse estudo tem como base mostrar como os cenários de simulação podem evitar erros e } \\
\text { melhorar a segurança dos pacientes em relação aos medicamentos. }\end{array}$ \\
\hline 5 & 2020 & Brasil & $\begin{array}{l}\text { Na perspectiva Histórico-cultural a simulação como instrumento psicológico contribuiu para o } \\
\text { desenvolvimento psíquico, mental, emocional dos acadêmicos para avaliarem e implementarem ações de } \\
\text { cuidado em cenários reais. Entretanto esse instrumento deve ser inserido articulado com fatores inter- } \\
\text { relacionados que se dão no campo sociocultural e que implicam diretamente com o desenvolvimento } \\
\text { humano e estão presentes no cotidiano do profissional. }\end{array}$ \\
\hline 6 & 2018 & $\begin{array}{l}\text { Arábia } \\
\text { Saudita }\end{array}$ & $\begin{array}{c}\text { O estudo indicou efeitos positivos no desenvolvimento do pensamento crítico e na autoconfiança dos } \\
\text { estudantes de enfermagem, causados tanto pelo ensino tradicional quanto pela Simulação Realística. O } \\
\text { estudo destaca, ainda, que não há uma diferença significante dos benefícios quanto aos métodos } \\
\text { comparados. }\end{array}$ \\
\hline 7 & 2016 & Canadá & $\begin{array}{l}\text { Diferença significativa demonstrada entre os grupos, com melhora no desempenho de competência, } \\
\text { julgamento clínico e experiência pré-briefing. }\end{array}$ \\
\hline 8 & 2016 & $\begin{array}{l}\text { Estados } \\
\text { Unidos }\end{array}$ & $\begin{array}{l}\text { Simulações de alta fidelidade são uma estratégia de aprendizagem eficaz e podem ser utilizadas no lugar de } \\
50 \% \text { das atividades tradicionais. }\end{array}$ \\
\hline
\end{tabular}

Fonte: Autores (2021).

Simular é um meio de aplicar a teoria lecionada nas aulas à prática vivenciada junto ao paciente. Os estudantes que participaram dos estudos apresentaram suas perspectivas quanto a metodologia ativa, as quais foram destacadas: a melhora na qualidade da assistência, a aquisição do controle do medo, o desenvolvimento da segurança, a eficácia do aprendizado quando comparado a metodologia passiva, a oportunidade de pensar acerca dos erros cometidos e o aperfeiçoamento de suas ações. A Simulação foi descrita pelos alunos como um instrumento de apoio ao desenvolvimento profissional levando os envolvidos a níveis mais elevados de raciocínio clínico. Entre os aspectos limitantes para a prática da simulação realística os artigos apresentaram: a exigência de recursos financeiros institucionais, problemas logísticos e de infraestrutura, adaptabilidade dos cenários, aquisição de simuladores, a necessidade de profissionais treinados para utilização dos equipamentos e nervosismo dos discentes.

Ao compararmos a perspectiva dos alunos expostos à prática realística com os não envolvidos nota-se grande diferença na absorção do conteúdo, principalmente os relacionados à SAE. Estudantes submetidos à simulação realística demonstram maior reconhecimento e capacidade de desenvolvimento da Sistematização da Assistência de Enfermagem. 


\section{Conclusão}

O estudo pôde concluir que os estudantes, após os cenários, estavam cientes de seus erros antes de receber o feedback de seus preceptores. Portanto, a simulação realística pode ser uma boa alternativa para facilitar a segurança, evitar erros e solidificar a aprendizagem da sistematização da assistência de enfermagem.

A participação em simulação de cenários de enfermagem antes de vivenciar a prática nos diversos serviços de saúde foi apresentada como uma oportunidade para o sucesso, visto que esta metodologia de ensino viabiliza um ambiente seguro para o treinamento de habilidades, desenvolvimento de pensamento clínico e a aprendizagem quanto a organização das suas ações, favorecendo o discente que ao ter contato com o paciente real saberá como agir sem nervosismo e com maior segurança, reduzindo erros. O uso da Simulação Realística demonstra ser uma forte ferramenta para a formação profissional pois funciona como auxílio para o controle das emoções, contribuindo assim para a preparação de alunos mais críticos, com destreza nas tomadas de decisões e atentos aos detalhes.

Findadas às análises bibliográficas tem-se que a percepção dos discentes acerca da aplicabilidade da Sistematização da Assistência de Enfermagem (SAE) no cenário de simulação realística é positiva, principalmente por contribuir no processo de intervenção.

Sugere-se aos estudos subsequentes, pesquisa qualiquantitativa, com grupo de estudantes delimitado, checklist para verificação de aprendizagem e validação de conteúdo, cenários de simulação com ênfase nos atributos necessários para aprendizagem e aprimoramento das habilidades clínicas para a execução as SAE.

\section{Referências}

Alamrani, M. H., Alammar, K. A., Alqahtani, S. S., \& Salem, O. A. (2018). Comparing the Effects of Simulation-Based and Traditional Teaching Methods on the Critical Thinking Abilities and Self-Confidence of Nursing Students. J Nurs Res 26(3):152-157. 10.1097/jnr.0000000000000231. https://pubmed.ncbi.nlm.nih.gov/29016466

Alves, N. P., Gomes, T. G., Lopes, M. M. C., Gubert, F. A., Lima, M. A., Beserra, E. P., Martins, M. C., \& Cavalcante, V. M. V. (2019). Simulação Realística e seus atributos para a formação do enfermeiro. Rev. enferm. UFPE on line 13(5): 1420-1428. https://pesquisa.bvsalud.org/portal/resource/pt/biblio-1024530

Cecchetto, F. H. \& Belaver, V. (2016). Evolução histórica da sistematização da assistência em enfermagem no Brasil. Revista Cuidado em EnfermagemCesuca. 2(2). http://ojs.cesuca.edu.br/index.php/revistaenfermagem/user

Bellaguarda, M. L. R., Knihs, N. S., Canever, B. P., Tholl, A. D., Alvarez, A. G., \& Teixeira, G. C. (2020). Simulação realística como ferramenta de ensino na comunicação de situação crítica em cuidados paliativos. Esc. Anna Nery 24(3). http://www.revenf.bvs.br/scielo.php?script=sci_arttext\&pid=S141481452020000300211

Chancey, R. J., Sampayo, E. M., Lemke, D. S., \& Doughty, C. B. (2018). L earners' Experiences During Rapid Cycle Deliberate Practice Simulations A Qualitative Analysis. Simulation in Healthcare: The Journal of the Society for Simulation in Healthcare: 14, 18-28 10.1097/SIH.0000000000000324. https://journals.lww.com/simulationinhealthcare/Fulltext/2019/02000/Learners_Experiences_During_Rapid_Cycle.3.aspx

Conselho Federal de Enfermagem (2009). Resolução $N^{o} 358$.

Cutrara, K. P. \& Turk, M. (2017). Impact of prebriefing on competency performance, clinical judgment and experience in simulation: An experimental study. Nurse Educ Today; 48:78-83. 10.1016/j.nedt.2016.09.012. https://pubmed.ncbi.nlm.nih.gov/27721089/

Ercole, F. F., Melo, L. S., \& Alcoforado, C. L. G. C. (2014). Revisão Integrativa versus Revisão Sistemática. Rev Min Enferm.; 18(1): 1-260. 10.5935/14152762.20140001. https://cdn.publisher.gn1.link/reme.org.br/pdf/v18n1a01.pdf

Ferenhof, H. A. \& Fernandes, R. F. (2016). Desmistificando a revisão de literatura como base para redação científica: Método SSF. Revista ACB: 21, 550-563. https://revista.acbsc.org.br/racb/article/view/1194

Ferreira, R. P. N., Helisamara, M. G., Dhelfeson, W. D. O., \& Miranda, J. L. (2018). Simulação Realística Como Método De Ensino No Aprendizado De Estudantes Da Área Da Saúde. Revista de Enfermagem do Centro-Oeste Mineiro 2018;8:e2508. 10.19175/recom.v7i0.2508 http://seer.ufsj.edu.br/index.php/recom/article/view/2508

George, J. B. (2000). Teorias de Enfermagem os Fundamentos à Prática Profissional. ARTMED (4a ed).

Gomes, R. G., Fava, S. M. C. L., Lima, R. S., Sanches, R. S., Gonçalves, M. F. C., \& Resck, Z. M. R. (2020). Desenvolvimento da competência de avaliação clínica do paciente crítico por acadêmicos de enfermagem: Contribuição da Simulação. Esc. Anna. Nery 24 (4). 10.1590/2177-9465-EAN-2019-0384 https://www.scielo.br/j/ean/a/XNnBrpYrMSsGRjfjQ6t54ZH/abstract/?lang=pt 
Research, Society and Development, v. 10, n. 9, e10110917711, 2021

(CC BY 4.0) | ISSN 2525-3409 | DOI: http://dx.doi.org/10.33448/rsd-v10i9.17711

Kaneko, R. M. U. \& Lopes, M. H. B. M. (2019). Cenário em simulação realística em saúde: o que é relevante para a sua elaboração? Rev. esc. enferm. USP 53. 10.1590/S1980-220X2018015703453. http://www.scielo.br/scielo.php?script=sci_arttext\&pid=S0080-62342019000100602\&lng=en\&nrm=iso

Kuo, S. Y., Wu, J. C., Chen, H. W., Chen, C. J., \& Hu, S. H. (2020). Comparison of the effects of simulation training and problem-based scenarios on the improvement of graduating nursing students to speak up about medication errors: A quasi-experimental study. Nurse Educ Today. Apr;87:104359. 10.1016/j.nedt.2020.104359. https://pubmed.ncbi.nlm.nih.gov/32058883/

Neco, K. K. S., Costa, R. A., \& Feijão, A. R. (2015). Sistematização da Assistência de Enfermagem em instituições de saúde no Brasil: revisão integrativa. Rev enferm UFPE on line., 9(1):193-200. https://periodicos.ufpe.br/revistas/revistaenfermagem/article/view/10325/11013

Klippel, C., Bastos, N., Camargo, E., Santos, H. A. S., Emmerick, L. G., Costa, L. C. R., \& Silva, R. C. L. (2020). Contribuição do debriefing no ensino baseado em simulação / Contribution of debriefing in simulation-based on simulation. Rev. enferm. UFPE on line; 14: [1-5]. https://pesquisa.bvsalud.org/brasil/resource/pt/biblio-1096020

Major, C. B., Mantovani, M. F., Felix, J. V. C., Boostel, R., Mattei, A. T., Arthur, J. P., \& Souza, R. M. (2020). Autoconfiança e satisfação dos estudantes de enfermagem em simulação de emergência. Rev Min Enferm. 2020;24:e-1336. 10.5935/1415.2762.20200073. http://reme.org.br/artigo/detalhes/1496

Meneses, A. R. C., Goiabeira, Y.N. L. A., Menezes, E. G., Lima, A. B. S., Jardim, M. J. A., \& Neto, M. L. (2019). Dificuldades dos acadêmicos de enfermagem na aplicabilidade da sistematização da assistência de enfermagem. Rev Fun Care Online; 11(1):181-185. 10.9789/2175-5361.2019.v11i1.181185. http://www.seer.unirio.br/index.php/cuidadofundamental/article/view/6879/pdf_1

Pereira, M. (2013). A seção de discussão de um artigo científico. Epidemiol. Serv. Saúde, 22(3):537-538. 10.5123/S1679-49742013000300020 http://scielo.iec.gov.br/pdf/ess/v22n3/v22n3a20.pdf

Pessanha, F. S., Barreto, B. M. F., Oliveira, B. G. R. B., Chrizostimo, M. M., Souza, D. F., \& Mafort, T. T. (2015). Principais microrganismos encontrados e produtos empregados em lesões tissulares contaminadas: revisão integrativa. OBJN, 14(1). 10.5935/1676-4285.20155111. http://www.objnursing.uff.br/index.php/nursing/article/view/5111

Rosa, M. E. C., Pereira, F. M. V., Góes, F. G. B., Caldeira, N. M. V., Souza, L. R. M., \& Goulart, M. C. L. (2020). Aspectos positivos e negativos da simulação clínica no ensino de enfermagem. Esc. Anna. Nery 24 (3). 10.1590/2177-9465-EAN-2019-0353 https://www.scielo.br/j/ean/a/w B9NcpWs3gnkgLm5JrjZ7zk/abstract/?lang=pt

Silva, J. L. G., \& Kumakura, A. R. S. (2018). Clinical simulation to teach nursing care for wounded patients. Rev. Bras. Enferm. 71(4). 10.1590/0034-71672017-0170. https://www.scielo.br/j/reben/a/HMGyyMKNQC5fknQphqtjTgj/?lang=en

Silva, T. A., Portella, P. C., Figueiredo, K. C., Wolff, L. D. G., \& Lima, E. F. (2021). Research, Society and Development, 10, e28210414200. 10.33448/rsdv10i4.14200. https://rsdjournal.org/index.php/rsd/article/view/14200/12682 